\title{
Figure S2
}

Intensity plots of rs 3797418 nd rs6082527. The plots are generated using Illumina Bead studio software. The plots show the data from Illumina 550K chip SNP assay.
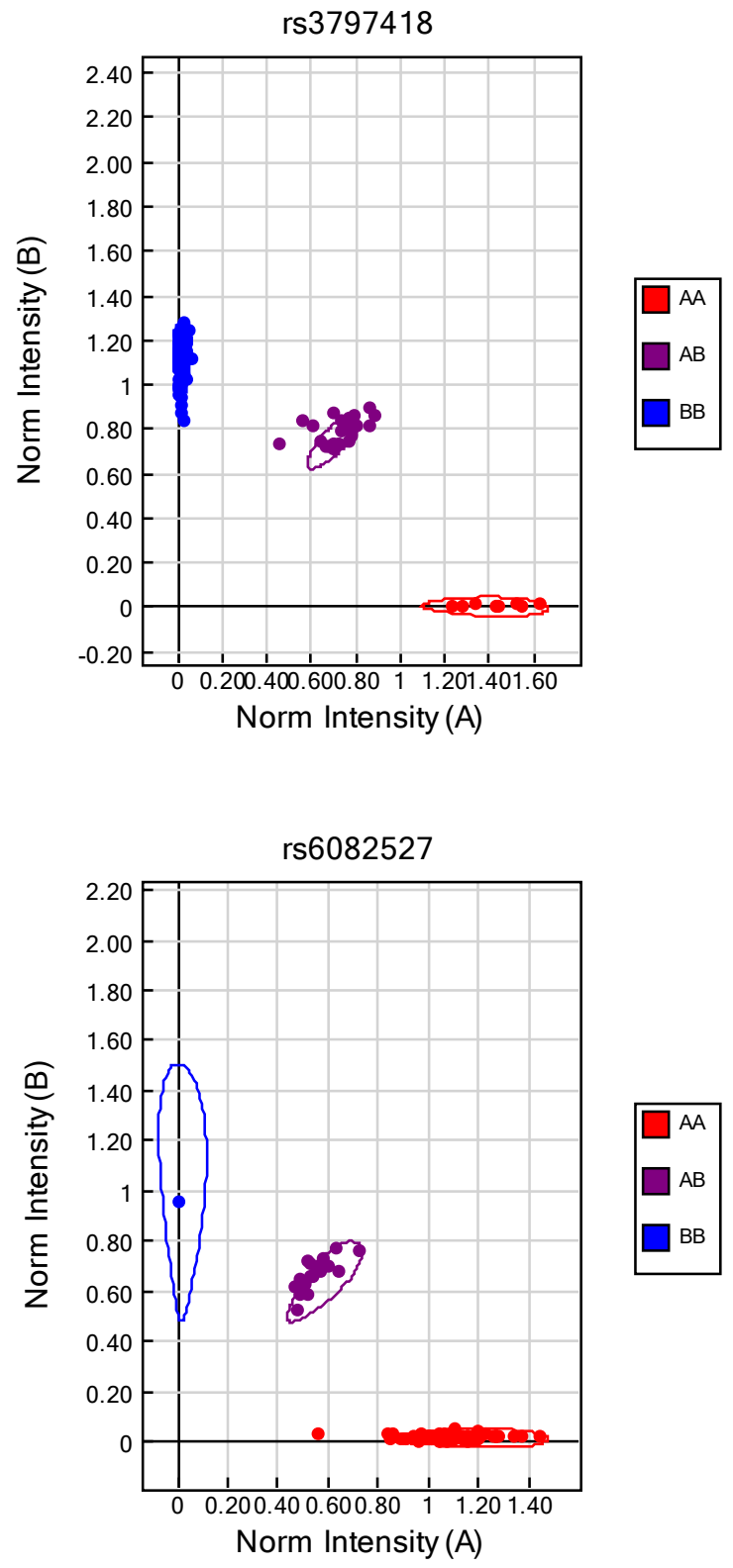
Intensity plots of top 5 SNPs from genome-wide SNP association study with gemcitabine IC $\mathbf{C}_{\mathbf{5 0}}$ value. The plots are generated using Illumina Bead studio software. The plots show the data from Illumina 550K chip SNP arrays.
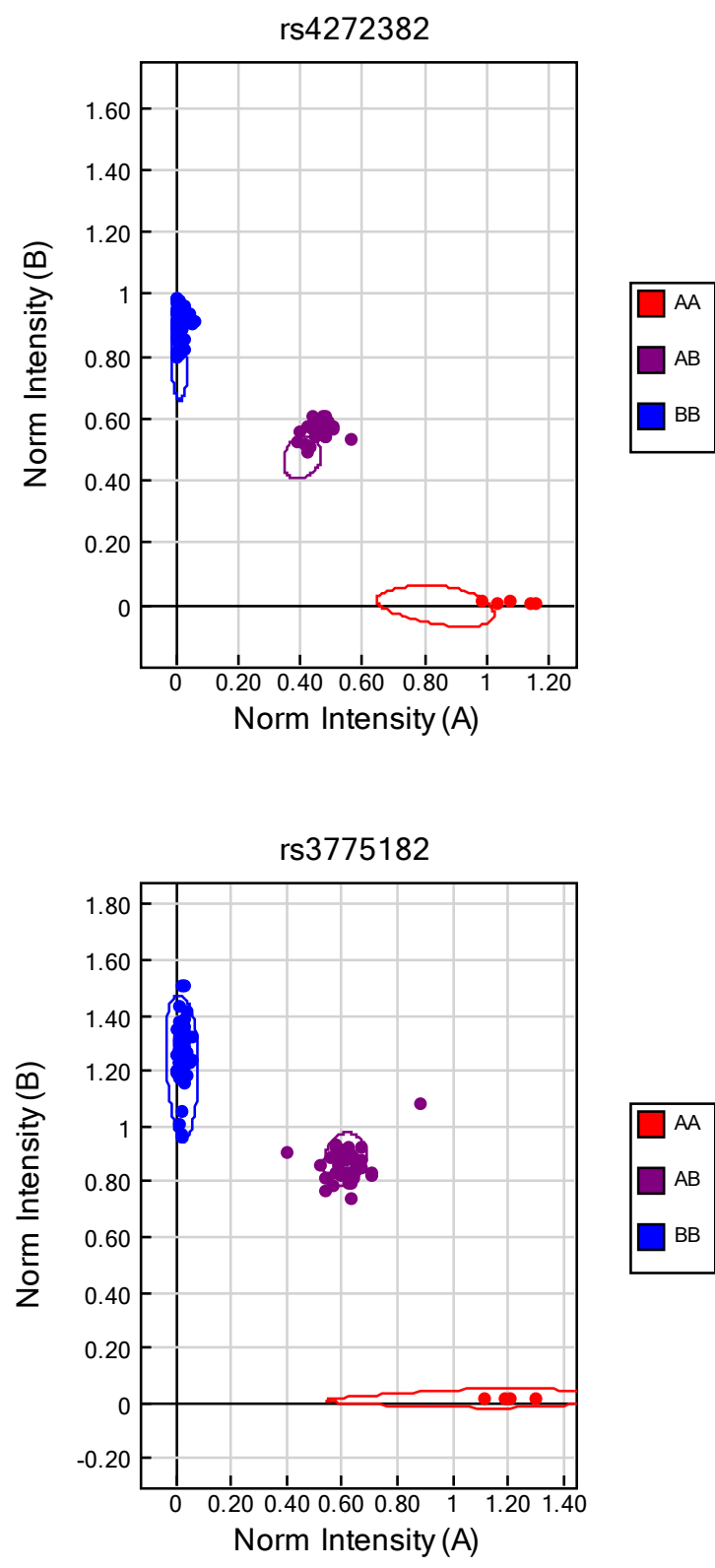

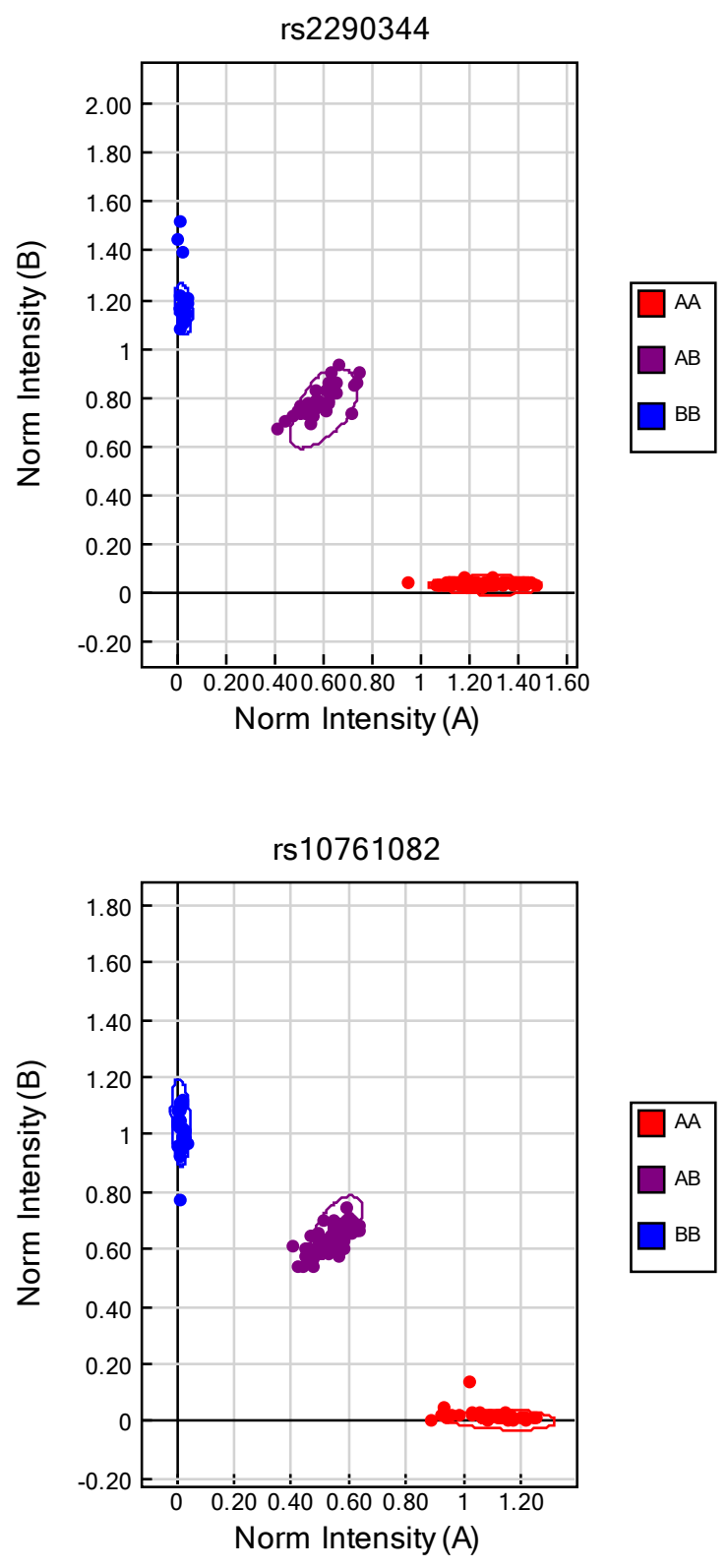


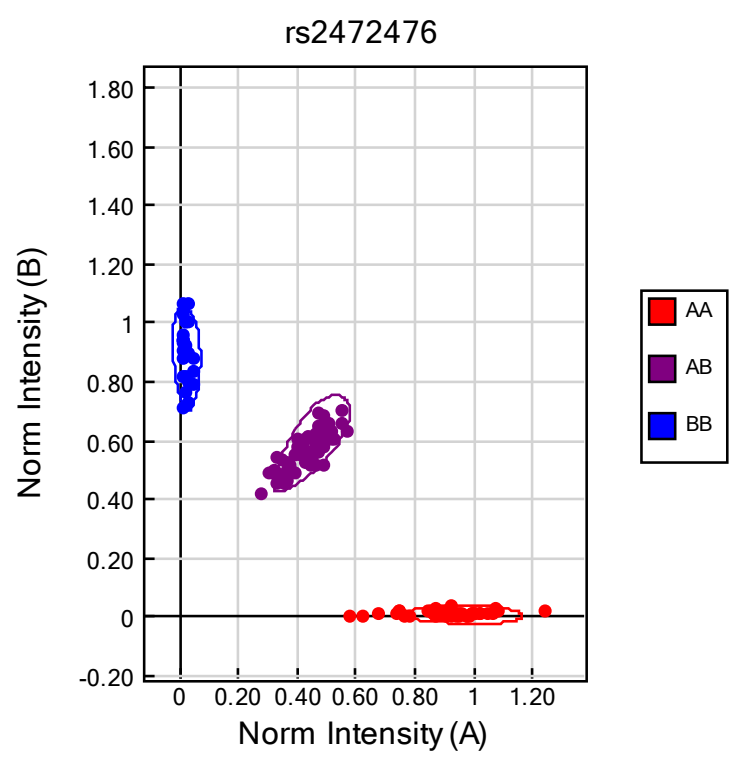

Intensity plots of top 5 SNPs from genome-wide SNP association study with AraC IC ${ }_{50} \underline{\text { value. The plots }}$ are generated using Illumina Bead studio software. The plots show the data from Illumina 550K chip SNP arrays.

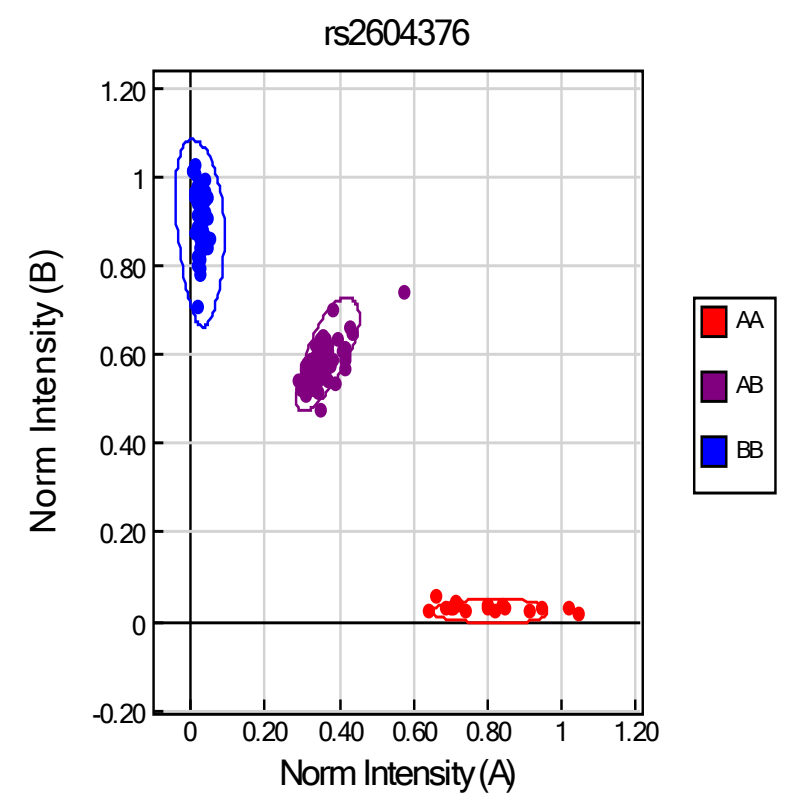



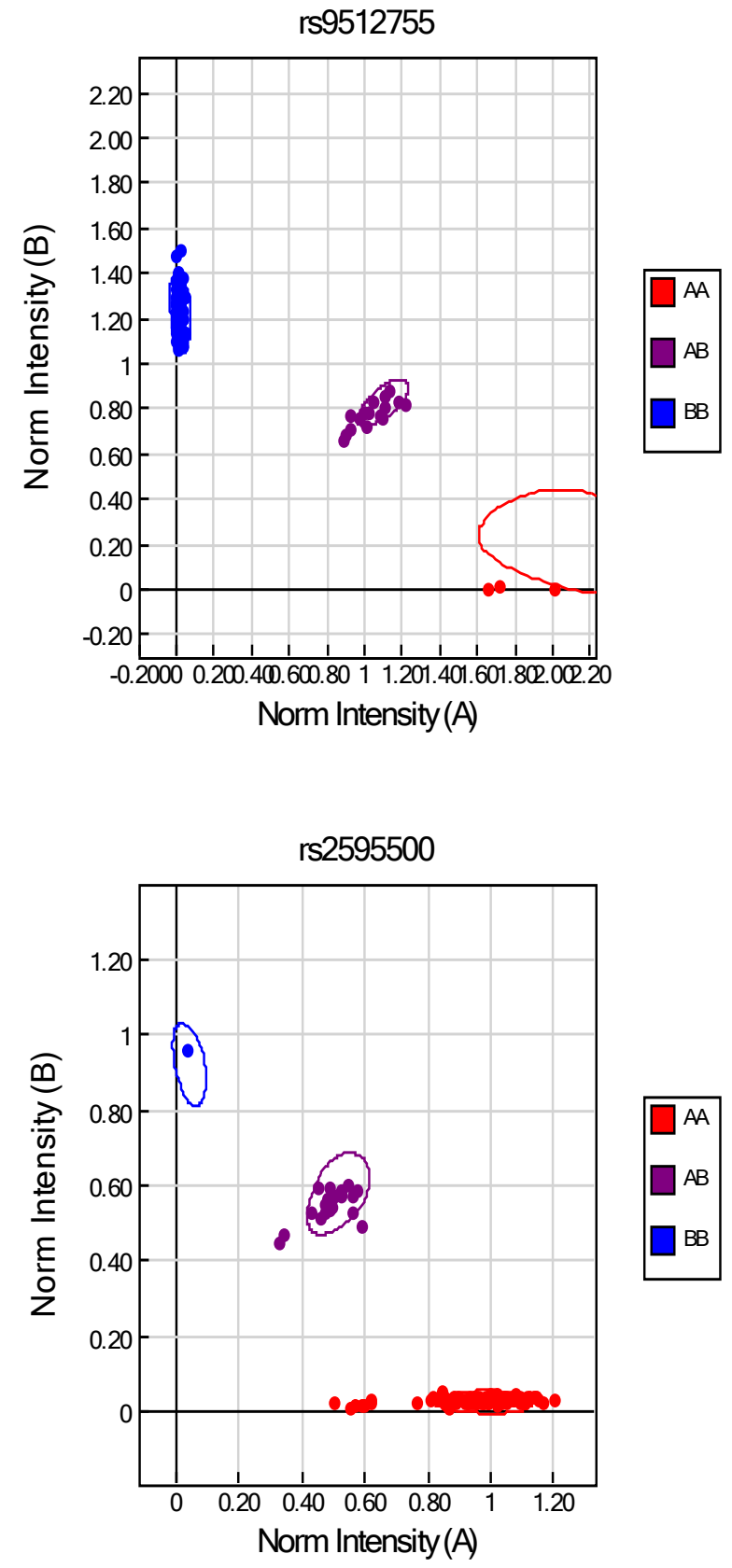

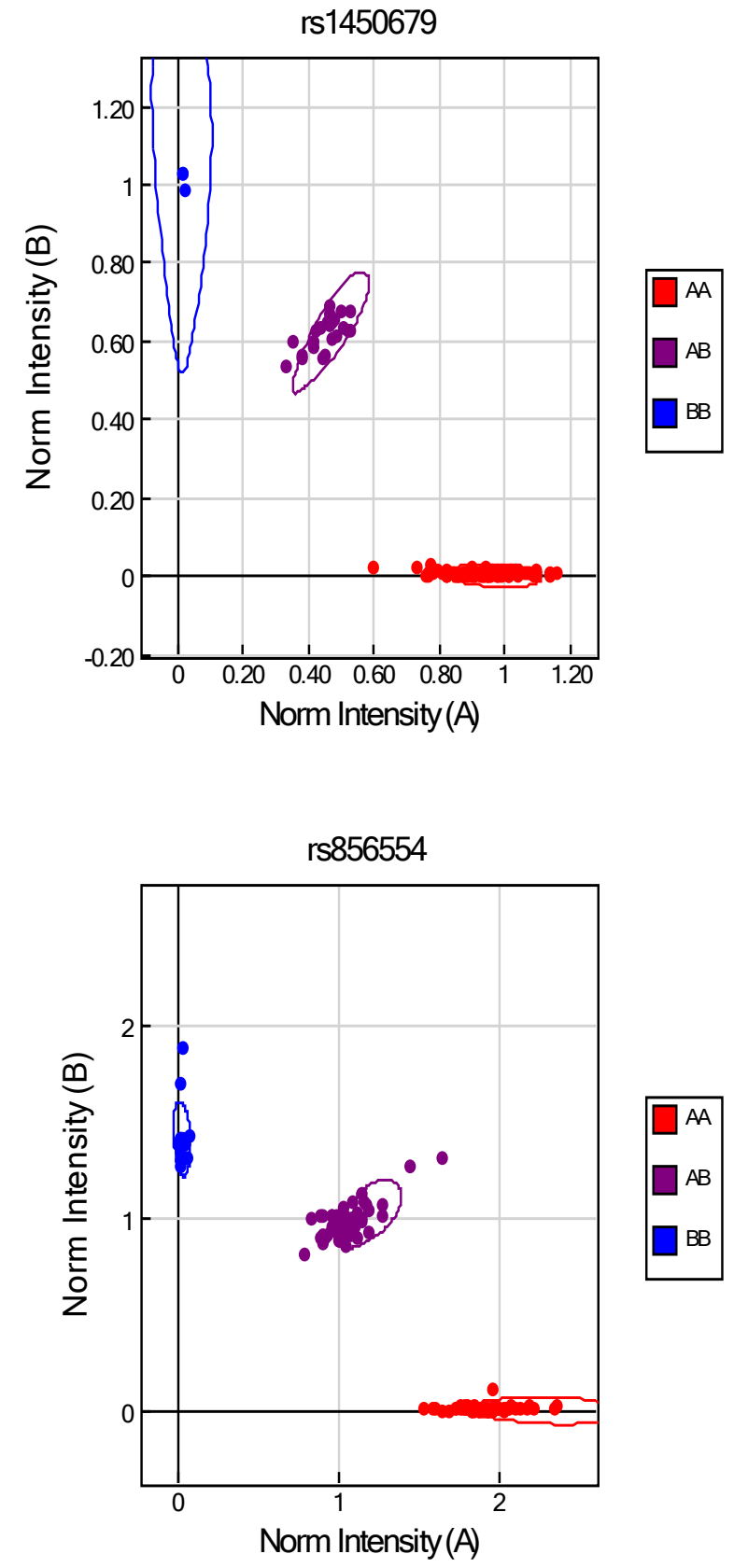